ARTICLE

\title{
Presence of low virulence chytrid fungi could protect European amphibians from more deadly strains
}

Mark S. Greener ${ }^{1}$, Elin Verbrugghe (10 1, Moira Kelly', Mark Blooi ${ }^{1}$, Wouter Beukema (i) ${ }^{1}$, Stefano Canessa (1) ${ }^{1}$, Salvador Carranza ${ }^{2}$, Siska Croubels $0^{3}$, Niels De Troyer ${ }^{4}$, Daniel Fernandez-Giberteau ${ }^{5}$, Peter Goethals ${ }^{4}$, Luc Lens ${ }^{6}$, Zhimin Li ${ }^{1}$, Gwij Stegen ${ }^{1}$, Diederik Strubbe ${ }^{6}$, Robby van Leeuwenberg${ }^{1}$, Sarah Van Praet ${ }^{1}$, Mireia Vila-Escale ${ }^{7}$, Muriel Vervaeke ${ }^{8}$, Frank Pasmans ${ }^{1} \&$ An Martel (iD) ${ }^{1 凶}$

Wildlife diseases are contributing to the current Earth's sixth mass extinction; one disease, chytridiomycosis, has caused mass amphibian die-offs. While global spread of a hypervirulent lineage of the fungus Batrachochytrium dendrobatidis (BdGPL) causes unprecedented loss of vertebrate diversity by decimating amphibian populations, its impact on amphibian communities is highly variable across regions. Here, we combine field data with in vitro and in vivo trials that demonstrate the presence of a markedly diverse variety of low virulence isolates of BdGPL in northern European amphibian communities. Pre-exposure to some of these low virulence isolates protects against disease following subsequent exposure to highly virulent $B d G P L$ in midwife toads (Alytes obstetricans) and alters infection dynamics of its sister species B. salamandrivorans in newts (Triturus marmoratus), but not in salamanders (Salamandra salamandra). The key role of pathogen virulence in the complex host-pathogenenvironment interaction supports efforts to limit pathogen pollution in a globalized world.

\footnotetext{
${ }^{1}$ Wildlife Health Ghent, Department of Pathology, Bacteriology and Avian Diseases, Faculty of Veterinary Medicine, Ghent University, B-9820 Merelbeke, Belgium. ${ }^{2}$ Institute of Evolutionary Biology (CSIC-UPF), 08003 Barcelona, Spain. ${ }^{3}$ Department of Pharmacology, Toxicology and Biochemistry, Faculty of Veterinary Medicine, Ghent University, B-9820 Merelbeke, Belgium. ${ }^{4}$ Department Animal Science and Aquatic Ecology, Faculty of Bioscience Engineering, Ghent University, Coupure Links 653, B-9000 Gent, Belgium. ${ }^{5}$ Centre de Recerca i Educacio Ambiental de Calafell (GRENP - Ajuntament de Calafell), 43882 Segur de Calafell, Spain. ${ }^{6}$ Terrestrial Ecology Unit, Department of Biology, Ghent University, K.L. Ledeganckstraat 35, 9000 Ghent, Belgium. ${ }^{7}$ Oficina Tècnica de Parcs Naturals, Diputació de Barcelona, 08036 Barcelona, Spain. 8 Agentschap voor Natuur en Bos, Havenlaan 88,1000 Brussel, Belgium.

凶email: An.Martel@ugent.be
} 
G lobal invasion by highly virulent chytrid fungi has resulted in the most extensive disease-driven loss of biodiversity ever recorded, with the decline of 500 amphibian species worldwide ${ }^{1,2}$. The skin disease chytridiomycosis is caused by two fungal species, Batrachochytrium dendrobatidis $(B d)^{3}$ and $B$. salamandrivorans $(\mathrm{Bsal})^{4}$. The vast majority of chytridiomycosisdriven declines have been attributed to the hypervirulent and panzootic lineage of $B d(B d G P L)^{1,5,6}$. Despite its presumed high virulence and global distribution, amphibian community declines caused by $B d \mathrm{GPL}$ are predominantly limited to disease hotspots in North, Central and South America, Australia, Europe and Africa $^{1,2,7-9}$. Since $B d$ GPL is also widely distributed in regions not affected by declines ${ }^{1}$, its mere presence is a poor predictor of disease dynamics. In Europe, BdGPL impacts vary from regional mortality events and declines in a limited number of species ${ }^{7,10-12}$ to widespread endemism characterized by host-pathogen coexistence in a broad range of host species ${ }^{1,2,13,14}$. Current knowledge of the host-pathogen-environment interaction ${ }^{15-22}$, notably the role of local $B d \mathrm{GPL}$ virulence types, offers an incomplete explanation of these different infections and disease dynamics.

Here we predict that $B d \mathrm{GPL}$ co-existence with amphibian communities is mediated by low virulence of the present $B d \mathrm{GPL}$ isolates and explore the extent to which low-virulence isolates confer protection against hypervirulent $B d$ or $B s a l$. We determined the occurrence of $B d \mathrm{GPL}$ in native amphibian populations in Belgium. We then compared pathogen virulence traits of northern European $B d \mathrm{GPL}$ isolates to those of a known hypervirulent isolate. Finally, we estimated the impact of pre-existing infections with less virulent $B d \mathrm{GPL}$ isolates on virulent $B d \mathrm{GPL}$ and Bsal infections.

\section{Results}

Populations persist in the presence of BdGPL. Opportunistic sampling of 1483 amphibians for $B d$ in 2015-2016 revealed the presence of $B d \mathrm{GPL}$ in 17 out of 63 locations and 5 out of 7 species sampled in Flanders, Belgium (Supplementary Fig. 1). Prevalence ranged between 0 and $35 \%$ per species (Supplementary Table 1). Besides, 25 cases of amphibian mortality, comprising 47 animals belonging to seven species (1 Rana temporaria, 20 Bufo bufo, 2 Triturus cristatus, 11 Ichthyosaura alpestris, 7 Lissotriton vulgaris, 3 Salamandra salamandra, 3 Lissotriton helveticus) were reported to the regional hotline (passive surveillance system) and submitted for postmortem analysis during a 4-year period (2015-2018). Chytridiomycosis was not diagnosed in any of these reported cases.

The impact of $B d$ on native amphibians was estimated both in remnant populations of a threatened chytridiomycosis susceptible species (midwife toads, Alytes obstetricans) and in populations of a common newt considered to be a $B d$ supershedder (alpine newt, Ichthyosaura alpestris). During the 4-year study, the midwife toad populations persisted in the presence of $B d$ infections (Supplementary Table 2). $B d$ occurrence was observed in 10 of 26 study populations of alpine newts and did neither result in reduction of newt abundance $(\mathrm{F}(2,76.2)=0.04, p=0.96)$ nor in reduction of body condition (i.e. scaled mass index, $\mathrm{F}(1,1728.13)=1.66, p=$ 0.20) (Supplementary Table 3, Supplementary Fig. 2).

Higher fecundity in hypervirulent $B d G P L$ compared to local $\boldsymbol{B d G P L}$ isolates. Ten $B d \mathrm{GPL}$ isolates were cultured from midwife toads (A. obstetricans), alpine newts (I. alpestris) or invasive bullfrogs (Lithobates catesbeianus) across Flanders, Belgium (Supplementary Table 4). These isolates were confirmed to belong to the GPL clade using a lineage-specific $\mathrm{PCR}^{23}$ and this was corroborated by whole-genome sequencing of 6 isolates ${ }^{1}$. Their culture phenotype (growth characteristics and morphological traits) was compared to that of the hypervirulent BdJEL423, isolated from an episode of amphibian mortality in the neotropics ${ }^{24}$. After 7 days of culturing, the hypervirulent BdJEL423 had significantly larger sporangia (linear model $\beta=0.28 \pm 0.02$, $t(10,416.8)=14.9, \quad P<0.001$; pairwise Tukey's HSD contrasts JEL423-other isolates $P<0.01$; Fig. 1) and produced significantly more zoospores (linear model $\beta=335.4 \pm 26.4, t(10,32)=12.72$, $P<0.001$; pairwise Tukey's HSD contrasts JEL423-other isolates $P<0.01$; Fig. 1) than any of the local $B d$ isolates (Fig. 1), corroborating previous reports that zoospore production and sporangium size are linked to pathogen virulence ${ }^{25,26}$. Although significant variation in phenotypic traits was also noticed among the local isolates, multivariate ordination of phenotypic traits grouped BdJEL423 apart from all other isolates (Fig. 1).

Midwife toads are a European sentinel species for $B d$-induced chytridiomycosis. To assess the potential impact of invasion of hypervirulent $B d \mathrm{GPL}$ in the broader amphibian community, we compared susceptibility of seven indigenous amphibian species to exposure to the hypervirulent $B d \mathrm{GPL}$ isolate $B d$ JEL423 and the local isolate $B d B E 1$. In contrast to the midwife toads that died after exposure to BdJEL423, European tree frogs (Hyla arborea), fire salamanders (Salamandra salamandra), spadefoot toads (Pelobates fuscus), alpine newts (I. alpestris) and natterjack toads (Epidalea calamita) became infected with both isolates (Supplementary Fig. 3) but did not develop lethal chytridiomycosis within the 13 weeks of the experiment. Throughout the trial, great crested newts (Triturus cristatus) and common toads (Bufo bufo) never tested positive for $B d$ (Supplementary Fig. 3). The marked susceptibility of midwife toads and the low susceptibility of fire salamanders and common toads correlate with marked fungal growth in mucosomes collected from midwife toads and with fungal growth inhibition by mucosomes collected from fire salamanders (Fig. 2a, Supplementary Fig. 4) and reflects the European field situation (endemic and widespread presence of $B d$, with focal outbreaks) $1,7,15,16,27-29$. Based on these results we classify the midwife toad as a $B d$ sentinel (canary in the coal mine for virulent $B d$ infections in Europe) species in Europe and decided to use this species in further experiments to test the pathogenicity of local isolates.

Midwife toads tolerate infection with local $B d G P L$ isolates but are highly susceptible to the hypervirulent $B d G P L$ isolate JEL423. Compared to the hypervirulent BdGPL isolate BdJEL423, all four local $B d G P L$ isolates included were low pathogenic in an infection trial using recently metamorphosed midwife toads (Alytes obstetricans) (Fig. 3). BdJEL423 caused 100\% mortality ( 9 out of 9 animals, housed individually) with a mean time to death after exposure of 27 days (range 16-64). Of the 29 toads infected with the local $B d G P L$ isolates, only one died due to infection. The probability of death was not significantly different from the uninfected control group (for penalized GLM coefficients, all $z$ $(5,43)<0.7, p>0.5$; Fig. 3, Supplementary Table 5).

BdGPL pathogenicity correlates with Crinkler and Necrosis (CRN) gene expression pattern and host cell invasion. Searching for pathogenicity determinants, the isolates were further compared by analysing the expression of a selection of $B d$ virulence genes in spores and spores that were first exposed to midwife toad skin. Regarding virulence gene expression, marked inter-isolate variation was noticed. Distinctive features mainly pertained to the expression of $\mathrm{CRN}$ and metalloprotease genes (Supplementary Tables 6-7). Expression of CRN genes has been linked to the early infection stage of $B d J E L 423^{5}$, and was low in spores of local $B d \mathrm{GPL}$ isolates ( $B d \mathrm{BE} 1, B d \mathrm{BE} 3, B d \mathrm{BE} 4$ and 

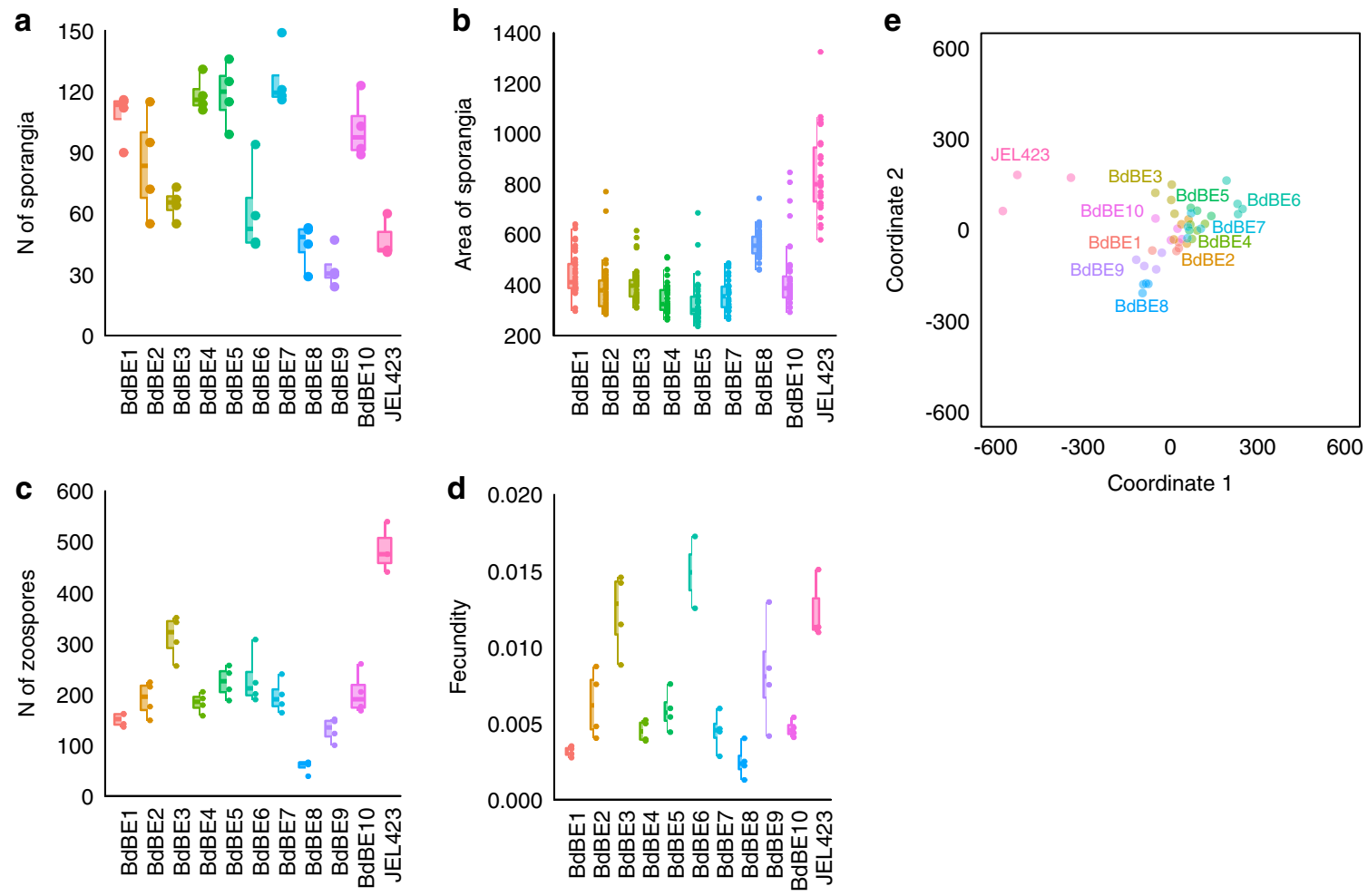

\begin{abstract}
Fig. 1 Experimental results for phenotypic traits of B. dendrobatidis isolates. a-d Boxes indicate 25th and 75th percentiles, central lines the mean, bars the 95 th percentiles, and points indicate individual samples. $\mathrm{N}$ of sporangia $=$ number of sporangia in the central $1000 \times 1000$ pixels, $n=4$ independent experiments; area of sporangia $=$ area of the largest 10 sporangia, $n=10$ technical replicates per independent experiment; $N$ of zoospores $=$ number of spores in the central $1000 \times 1000$ pixels of the well/image, $n=4$ independent experiments; fecundity was calculated following the formula (average $\mathrm{N}_{\text {ZOOSPORE}}$ /average $\mathrm{N}_{\mathrm{SPOR}}$ )/average $\mathrm{A}_{\mathrm{SPOR}}, n=4$ independent experiments. e Non-metric multidimensional scaling across the four variables in (a-d); labels indicate one point per group as a colour legend. BdBE1-10 are local isolates isolated in this study. JEL423 is a BdGPL isolate isolated from an episode of amphibian mortality in the neotropics ${ }^{16}$. Source data are provided as a Source data file.
\end{abstract}

$B d B E 5)$ compared to the hypervirulent $B d$ JEL423. Incubation with midwife toad tissue resulted in increased CRN gene expression for isolates $B d$ JEL423, BdBE1 and $B d B E 3$ and decreased expression for $B d B E 4$ and $B d B E 5$ (Fig. 2b, Supplementary Fig. 5, Supplementary Tables 6-7). In vitro, this pattern of CRN gene expression was correlated with invasiveness and intracellular growth in amphibian A6 cells. Intracellular growth, characterized by the formation of intracellular daughter thalli, was only observed for $B d$ JEL423, $B d \mathrm{BE} 1$ and $B d \mathrm{BE} 3$. Growth of $B d B E 4$ and $B d B E 5$ was dominated by an epibiotic growth, limited to $B d$ development outside the host cells (Fig. 2d, e, Supplementary Figs. 6-8). Relative CRN gene expression followed the same pattern as colonisation capacity in the above mentioned in vivo infection experiment, with significant correlation between CRN gene expression of fresh spores of local BdGPL isolates relative to $B d$ JEL423 and skin colonisation capacity (CRN_22492 $\left.r_{\mathrm{s}}=0.88, p \leq 0.05, n=38\right)\left(\mathrm{CRN} \_25085 r_{\mathrm{s}}=0.73, p \leq 0.05, n=\right.$ 38) $\left(\mathrm{CRN} \_23176 r_{\mathrm{s}}=0.88, p \leq 0.05, n=38\right)^{5}$.

Genome-wide variance associated with BdGPL virulence and host cell invasion. Whole-genome sequence data were mapped against the reference genome for BdJEL423 to identify genomewide variation associated with the low virulence $(B d \mathrm{BE} 1, B d \mathrm{BE} 3$, $B d \mathrm{BE} 4$ and $B d \mathrm{BE} 5)$ and cell invasion (BdBE1, $B d \mathrm{BE} 3$ and $B d$ JEL423) phenotypes. Annotation of variants predicted to be highly deleterious or disruptive to gene function predicted 101 genes presenting differentiating variants between local isolates and the hypervirulent $B d$ JEL423 isolate and 64 genes differentially affected between isolates displaying the invasive versus the epibiotic phenotype. Many of these genes were annotated as candidate Crinkler genes (12 genes), or contained common effector protein domains such as SigP4 secretory signals (28 genes), transmembrane domains (10 genes), or metalloprotease (4 genes), aspartate proteases (12 genes) and CBM18 chitin-binding PFAM domains (4 genes, Supplementary Datasheet 1). Fisher's exact test indicated gene enrichment of five PFAM domains in both sets of affected genes, including Aspartate proteases, the chitin-binding CBM18 domain and the M36 metalloproteaseassociated FTP domain (Supplementary Datasheet 1). Three of the genes affected by deleterious mutations in all local isolates show upregulated expression in BdJEL423 in vivo ${ }^{5}$, including BDEG_22285, a secreted M36 metallopeptidase. The established role of Crinkler proteins ${ }^{30}$, M36 metalloproteases $^{31,32}$ and aspartate protease s $^{33-35}$ in fungal pathogenesis suggest these may be $B d$ effector proteins that are differentially associated with function-altering mutations in virulent and invasive phenotypes, and highlights targets for future investigation. As previous studies have found pathogen virulence attenuation in BdGPL to be associated with an overall decrease in copy number ${ }^{24}$, this was compared in the local and BdJEL423 isolates. While the local isolates showed extensive copy number variation of large sequence regions up to entire supercontigs, no such overall decrease in copy number was observed in the local isolates compared with BdJEL423 (Supplementary Fig. 9).

Local $B d G P L$ protects midwife toads against hypervirulent $\boldsymbol{B d G P L}$. To assess the level of protection conferred by the local $B d \mathrm{GPL}$ isolates against invasion by hypervirulent $B d \mathrm{GPL}$, we exposed the animals that were previously infected with one of four local BdGPL isolates to the hypervirulent BdJEL423 and 
a

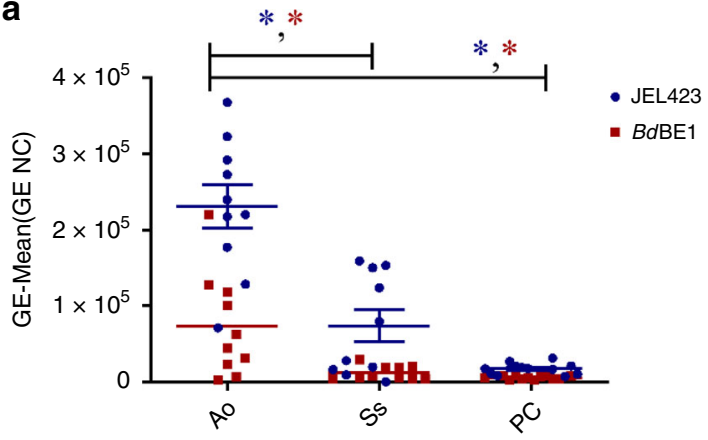

b

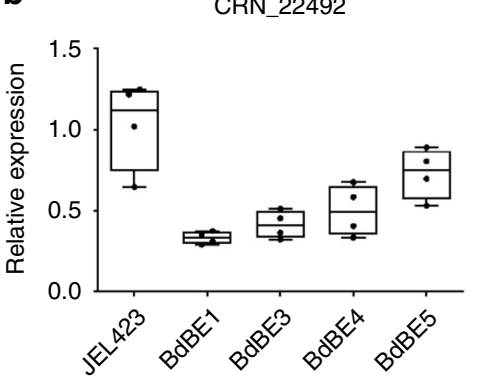

CRN_25085

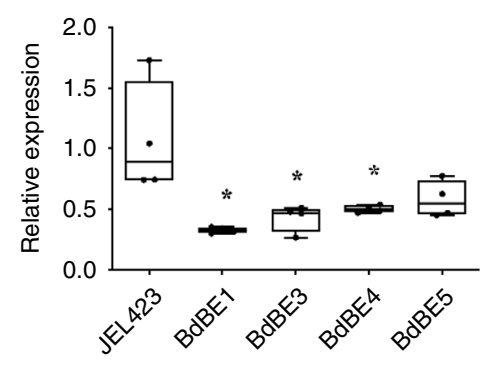

CRN_23176

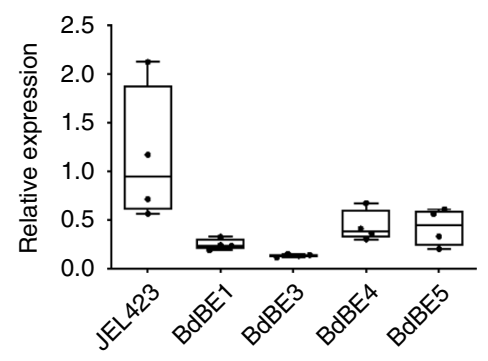

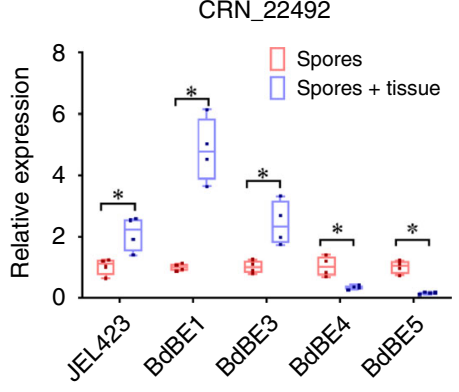

CRN_25085

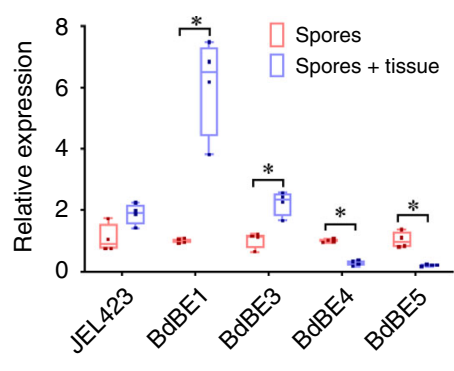

CRN_23176

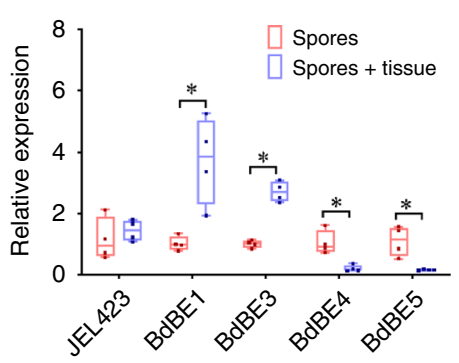

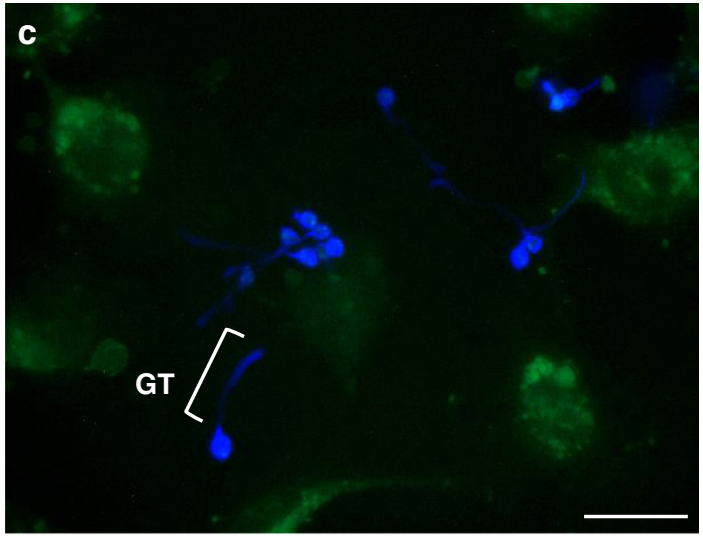
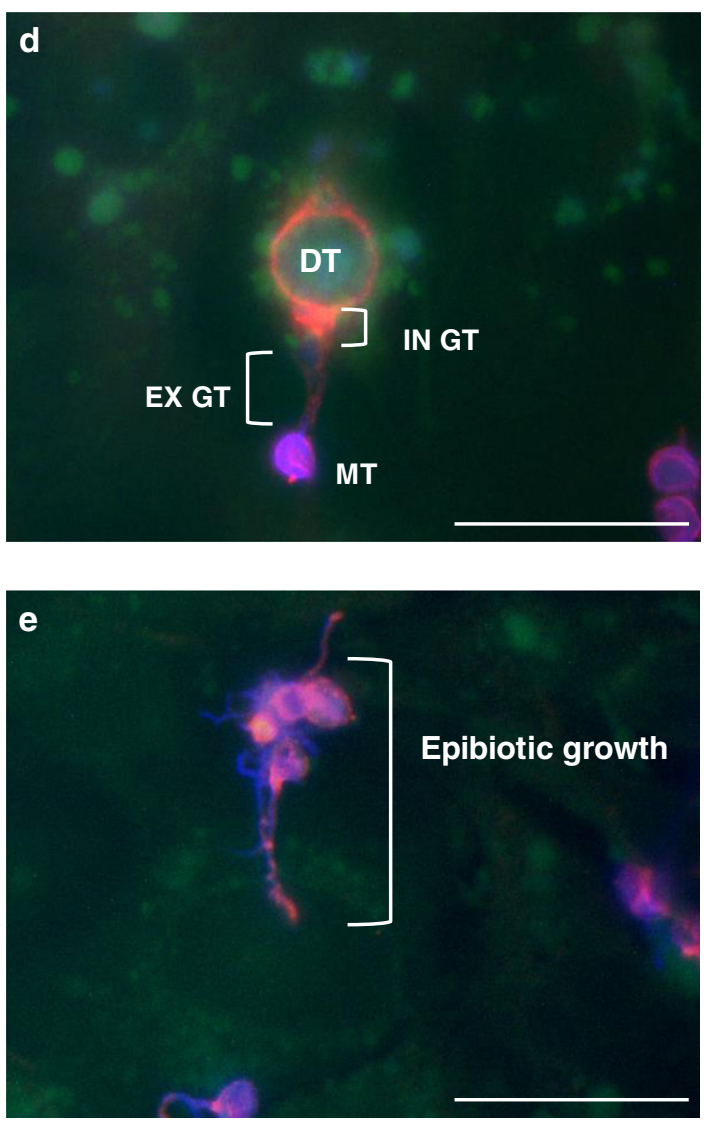

compared infection dynamics and disease with that of age-matched, naive toads exposed to BdJEL423 only. Animals that had been previously infected with the $B d \mathrm{BE} 1$ and $B d \mathrm{BE} 3$ isolate showed significantly higher survival than those infected directly with $B d$ JEL423 (GLM $\beta=-2.59 \pm 1.29, z(2,32)=-2.01, p=$ 0.044; Fig. 3, Supplementary Table 8).

In a final proof of concept study, infection dynamics of the hypervirulent $B d$ JEL423 were compared between midwife toadlets that were either pre-exposed or not pre-exposed (naive) to lowvirulence $B d \mathrm{BE} 3 \quad(n=20$ per treatment, all animals kept individually) 4 weeks before BdJEL423 challenge. Exposure to $B d B E 3$ resulted in infection in all animals in the absence of mortality and a median peak load of 140 GE (range 14-1164 GE). Subsequent challenge of these pre-exposed toads with $B d$ JEL423 resulted in detection of low $B d$ levels in only two toadlets ( 3 and $23 \mathrm{GE})$ and only on the first sampling occasion. In contrast, all naive animals developed significant infection loads during the 4- week follow-up period after challenge with $B d$ JEL423 (median peak load 1802 GE, range 58-19,100 GE) (Fig. 3). Prior infection with the low-virulence $B d B E 3$ thus resulted in less infections upon challenge with the highly virulent BdJEL423 (Fisher's exact test, two-tailed $P$ value $<0.0001)$.

$B d G P L$ alters virulent $B s a l$ infection in marbled newts, but not fire salamanders. Local $B d \mathrm{GPL}$ temporarily colonized fire salamanders (S. salamandra), marbled newts (Triturus marmoratus) and ribbed newts (Pleurodeles waltl) without causing clinical signs or mortality (Fig. 4). The effect of pre-exposure to local BdGPL on the course of a subsequent Bsal infection depended on the infected species (Fig. 4, Supplementary Table 9). In fire salamanders, exposure to local BdGPL had no effect on Bsal-induced mortality (penalized GLM $\beta=0.00 \pm 2.17, z(2,26)=0.00, p=1$ ) or infection course (parametric survival regression $\beta=-0.07 \pm$ 
Fig. 2 BdGPL virulence coincides with growth ability in mucosomes, Crinkler (CRN) gene expression and adhesion and invasion capacities. a Growth (expressed in corrected GE values) of BdBE1 and BdJEL423 in mucosomes collected from different amphibian species. PC: positive control; Ao: Alytes obstetricans; Ss: Salamandra salamandra; NC: negative control. Significant differences are shown with an asterisk and were assessed by a Kruskal-Wallis analysis, followed by pairwise Mann-Whitney U-test (two-tailed) with a Bonferroni-corrected $P$ value of 0.017 (Ao vs Ss: $P$ value $=0.001$ (BdJEL423) and 0.009 (BdBE1); Ao vs PC: $P$ value $<0.001$ (BdJEL423) and 0.003 (BdBE1)). The experiment was carried out in tenfold and individual data points are shown (blue $=B d J E L 423$; red $=B d B E 1$ ), with the mean \pm s.e.m. depicted by the horizontal bars. $\mathbf{b}$ Box plots of mean fold changes in mRNA expression of CRN genes (CRN_22492, CRN_25085 and CRN_23176). The data in the left panel show the normalized target gene amount in spores of each isolate ( $n=4)$ relative to spores of $B d J E L 423(n=4)$ which is considered 1. The data in the right panel show the normalized target gene amount in spores that were incubated with skin tissue of $A$. obstetricans for $2 \mathrm{~h}(n=4)$ relative to spores of the respective isolate $(n=4)$ which is considered 1 . Boxes indicate 25 th and 75th percentiles, central lines the median, bars the minima and maxima, and points indicate individual samples. Target genes were based on Farrer et al. ${ }^{5}$. Left panel: an asterisk indicates a significant difference compared to BdJEL423 spores (Kruskal-Wallis analysis, followed by pairwise Mann-Whitney Utests (two-tailed) with a Benjamini-Hochberg-adjusted $P$ value $<0.05$ ). Right panel: an asterisk indicates a significant difference compared to spores of the respective isolate (Kruskal-Wallis analysis, followed by pairwise Mann-Whitney U-tests (two-tailed). Individual $P$ values are shown in Supplementary Tables 6-7. c Representative image showing germ tube formation of BdJEL423. Fluorescent signals of Bd (Calcofluor White (blue)) and A6 cells (green cell tracker) were merged to assess the ability of germ tube formation, $4 \mathrm{~h}$ after contact with the cells. $\mathbf{d}$, e Representative images demonstrating endobiotic growth (d: BdJEL423) and epibiotic growth (e: BdBE4) of Bd. The cell wall of extracellular $B d$ was coloured using Calcofluor White (blue). $B d$ was visualized using Alexa Fluor 568 targeting a polyclonal antibody against $B d$ (Thomas et al. ${ }^{62}$ ), resulting in red fluorescence of both intracellular and extracellular $B d$. The $B d$-exposed A6 cells were stained with a cell tracker (green). $\mathbf{d}$ New intracellular chytrid thalli are formed and the cell content of the mother thallus is transferred into the new daughter thallus (DT). e Epibiotic growth is limited to Bd development outside the host cells. GT germ tube, EX GT extracellular germ tube, IN GT intracellular germ tube, MT mother thallus, DT daughter thallus. Scale bar $=20 \mu \mathrm{m}$. Three independent in vitro experiments were conducted with every condition being tested in triplicate, with similar results. Source data are provided as a Source data file.

a

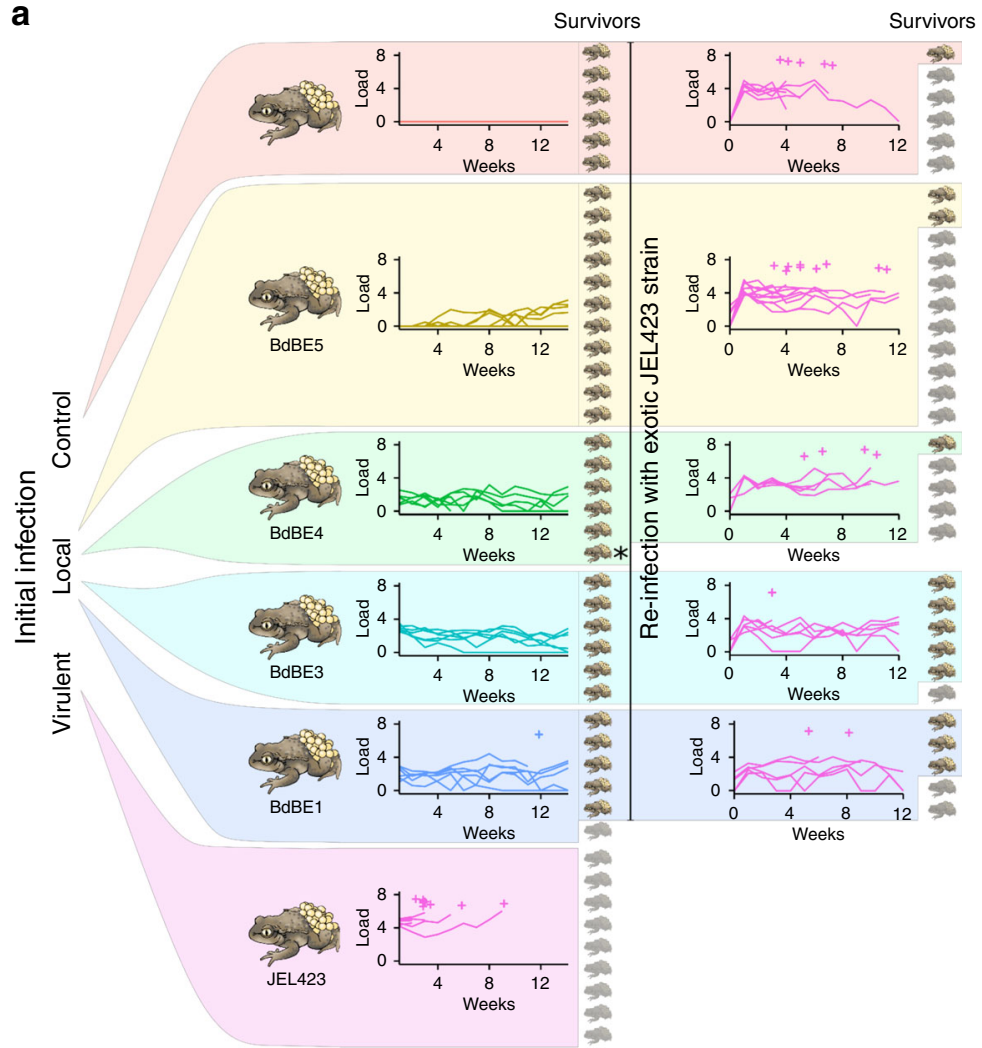

b

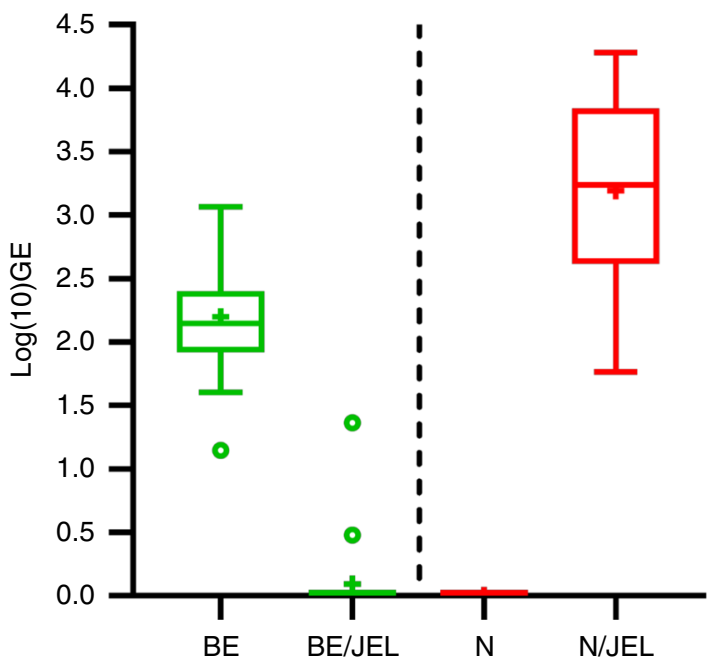

Fig. 3 Local BdGPL isolates protect midwife toads against infection with virulent BdGPL. a (Left) Toads exposed to local (BdBE) isolates had survival comparable to the control (non-exposed) group; toads exposed to hypervirulent BdJEL423 had $100 \%$ mortality. (Right) When all survivors were re-infected with $B d J E L 423$, toads previously exposed to BdBE1 and BdBE3 had higher survival than naive toads (control) or toads previously exposed to BdBE4-5. For each group, curves indicate infection load $\left(\log _{10}[G E+1]\right.$ ), crosses indicate times of individual deaths (crosses offset vertically for readability, dead individuals shaded). The asterisk * indicates one animal that died of non-treatment-related causes between the two experiments. $\mathbf{b}$ Tukey-style box plots of $B d$ peak loads in midwife toads either pre-exposed (BE: green) or not pre-exposed ( $N$ : red) to the low-virulence BdBE3 isolate and 4 weeks later challenged with the highly virulent BdJEL423 (BE/JEL and N/JEL). $n=20$ biologically independent animals per condition. Source data are provided as a Source data file. 

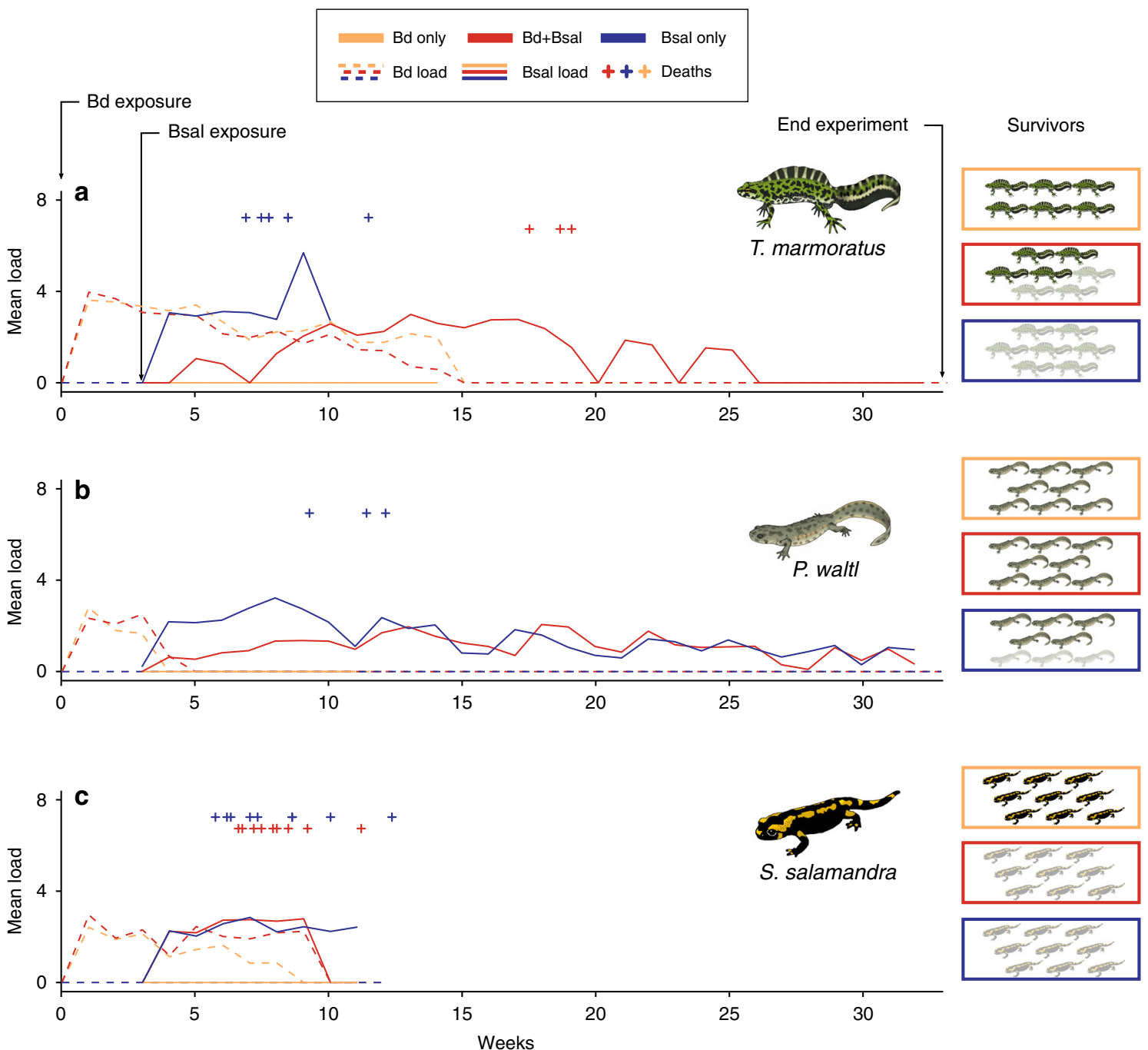

Weeks

Fig. 4 Low-virulence BdGPL isolates protect some urodelan species, but not others, against infection with B. salamandrivorans. a For Triturus marmoratus, low virulent BdGPL exposure reduced Bsal-induced mortality but increased the length of the infectious period. b For Pleurodeles waltl, lowvirulence BdGPL exposure reduced Bsal-induced mortality but had no effect on the length of infectious period or on infection loads. c For Salamandra salamandra, low virulent BdGPL exposure had no effect on Bsal-induced mortality or infection course. For each species, Bsal exposure occurred 21 days after $B d$ exposure. For all groups, curves indicate infection load $\left(\log _{10}[G E+1]\right)$, crosses the time of individual deaths (offset vertically for readability), boxes the number of survivors in each treatment group by species (dead individuals shaded). Source data are provided as a Source data file.

$0.15, z(1,18)=-0.43, p=0.67)$; Bsal exposure always resulted in lethal infection at an average of 35 days for both treatments. In ribbed newts, $B d \mathrm{GPL}$ exposure did not significantly change mortality (penalized GLM $\beta=0.00 \pm 2.18, z(2,23)=0.00, p=1$ ) or the duration of the infection period (model could not be fitted; Fig. $4 \mathrm{~b}$ ), yet, mortality was observed in the non- $B d$ pre-exposed newts only. All marbled newts that were inoculated with Bsal only died within an average of 37 days after exposure, whereas four out of seven newts survived when pre-exposed to $B d G P L$ (penalized GLM $\beta=2.95 \pm 1.74, z(2,19)=1.71, p=0.088$, one-sided $t$-test, $p=0.037$ ). However, $B d$ GPL pre-exposure increased the duration of the infectious period (parametric survival regression $\beta=1.49$ $\pm 0.21, z(1,14)=7.14, P<0.001)$ and it took on average 107 days before three of the newts died.

\section{Discussion}

Although $B d$ GPL has caused death and species extinction on a global scale, its impact in Europe is currently limited to specific regions. Our results suggest that low-virulence $B d \mathrm{GPL}$ isolates co- occur with amphibian communities in the absence of population declines and that only a limited number of European amphibian species may be susceptible to the highly virulent isolates associated with massive declines elsewhere. Results of our laboratory trials with seven indigenous amphibian species largely match the in situ condition in northern Europe $e^{1,13,15,27-29}$. None of the tested species was susceptible to $B d$-induced chytridiomycosis after exposure to the local BdGPL isolates but several showed infection patterns that corroborate their potential as a chytrid reservoir. Midwife toads persisted in small and isolated populations during at least 4 years in the presence of $B d$ and $B d$ infection could not be linked to decreased body condition or host abundance in alpine newts. This finding corroborates the current coexistence of low-virulence BdGPL with northern European amphibian assemblages, despite the occurrence of erratic mortality ${ }^{13,36}$.

In contrast, the hypervirulent $B d \mathrm{GPL}$ isolate did cause lethal infections in a single amphibian species, the midwife toad, while several other species showed relatively high-level long-term infections. These experimental results agree with $B d$ epizootics 
and population declines observed in midwife toads in Southern Europe ${ }^{7,10-12,37}$

Our results suggest that if a hypervirulent BdGPL similar to the one used in this study invaded our study area, two scenarios might occur. If the invaded host population is entirely $B d$-naive, susceptible hosts (e.g. midwife toads) are likely to be lethally affected. Co-occurring, tolerant hosts (e.g. alpine newts) may sustain highlevel infections and act as pathogen reservoirs. If spatio-temporal factors shape a conducive environment, these disease dynamics might result in mass die-offs, population declines and even extirpation of the susceptible species ${ }^{38}$. However, pre-existing lowvirulence $B d$ isolates may at least initially affect disease dynamics of an invading hypervirulent $B d$. Their protective effect against disease is associated with several traits that correlate with their colonisation ability and invasiveness: expression and function of $B d$ crinkler genes, metalloproteases and other effector proteins. However, the hypervirulent isolate's high fecundity, marked invasiveness in amphibian cells and more pronounced colonization capacity in vivo suggest a distinct competitive advantage over the local isolates ${ }^{39}$. Moreover, co-infections may result in superinfection and/or hybridisation ${ }^{3,40,41}$, with highly unpredictable outcomes.

Reduced mortality and delayed time to death demonstrate that prior exposure to $B d$ reduces pathogenicity of $B s a l$ in marbled newts. This may equally pertain to the ribbed newts, in which Bsal mortality occurred only in newts that were not previously exposed to $B d$. However, low mortality rate and relatively small sample size hamper meaningful statistics for this species. In contrast, all fire salamanders succumbed to Bsal infection within a similar timeframe, regardless of prior $B d$ infection. The host species-dependent protective effect of a pre-existing $B d$ infection against Bsal may present as a double-edged sword for the amphibian community. The reduced pathogenicity in the marbled newts coincides with a longer infectious period and high Bsal infection loads, which is likely to facilitate Bsal transmission to susceptible animals. Increased transmission opportunities may offer an additional explanation for the local extirpations observed in Bsal-infected fire salamander populations in parts of Europe where $B d$ is widespread ${ }^{4,42}$. In amphibian communities, simultaneous introduction of both pathogens may be subject to different disease dynamics. Simultaneous co-infections with $B d$ and Bsal in American newts (Notophthalmus viridescens) resulted in disease exacerbation ${ }^{43}$.

In conclusion, our results show a complex disease landscape with multiple implications for biodiversity conservation. First, the mere demonstration of $B d G P L$ presence in amphibian communities is not sufficient to predict disease impacts on susceptible species. Second, co-existence of amphibian communities with low-virulence BdGPL may alter the outcome of an invasion by a hypervirulent isolate ${ }^{44}$. Protection may increase the probability of host populations surviving a hypervirulent chytrid incursion, but also amplify reservoir dynamics and increase the risk to highly susceptible species. European mitigation programmes could be fine-tuned to focus on the highly virulent isolates, but this would require the availability of a rapid diagnostic pathogen virulence assay.

\footnotetext{
Methods

Impact of Bd endemism in amphibian communities in Flanders. In a first study, $B d$ prevalence was determined across our study area (Flanders, Belgium). We sampled 1483 amphibians belonging to 62 populations in 2015-2016 (Supplementary Fig. 1). To detect the presence of $B d$, we collected swabs from the superficial skin surface of metamorphosed animals or the mouthparts of larval anurans. To study potential co-existence of $B d$ in the study region with small populations of a susceptible species (where negative effects are expected to be most obvious), in a second study, we sampled five breeding sites of midwife toads in Flanders for 4
} consecutive years (Supplementary Table 2). In these breeding sites, larvae were counted once a year and their mouthparts were sampled for the presence of $B d$. In a third field study, we selected 26 ponds across our study area containing at least a population of alpine newt (Ichthyosaura alpestris), being the European urodele most likely infected by $B d$. Ponds were sampled with funnel traps three or four times (depending on the presence of water) with a 1-month interval (March-June 2019). An envisaged 30 newts per sampling per pond were swabbed for the presence of $B d$ (Supplementary Table 3), weighed to the nearest $0.1 \mathrm{~g}$ and the snout-vent length measured to the nearest $\mathrm{mm}$. As an estimate of body condition, we used the scaled mass index $(\mathrm{SMI})^{45}$, which adjusts the mass of all individuals to that which they would have obtained if they had the same body size. SMI was calculated using the equation of the linear regression of log-body mass on log-snout-vent length estimated by type 2 (standardized major axis; SMA) regression ${ }^{45}$. Eleven outliers were present (i.e. |standardized residual $>3$ ). These observations were not used for deriving SMI relationships (as per Peig and Green ${ }^{45}$ ). The regression slope was 2.87, and average snout-vent length was $42.7 \mathrm{~mm}$. We thus calculated the SMI as (body mass $\left.\times(42.7 / \text { snout-vent length })^{2.87}\right)$. The average number of individuals caught per fyke per pond was used as proxy for newt density. To test whether trends in newt density (i.e. average number of newts per fyke) differed between $B d$ positive and $B d$ negative ponds, a generalized linear mixed model (GLMM) was used specifying newt density as the dependent variable and the interaction between time (month) and $B d$ status (positive versus negative) as independent variables. Trends in newt density were better approximated by a quadratic relationship compared to a linear trend (delta AIC $=5.73$ ). To test whether trends in SMI differed between $B d$ positive versus $B d$ negative newts, a GLMM was implemented using SMI as the dependent variable and time (month), $B d$ status (positive versus negative), newt sex (male versus female) and newt density (see above) as independent variables. The initial model contained all two-way interactions between the independent variables. For both GLMMs, pond was implemented as a random factor, and a Gaussian error structure was specified (model residuals were normally distributed, Shapiro-Wilk $W>0.90$ ). A frequentist approach was adopted whereby initial models were reduced in a stepwise manner, by excluding the variable with the highest $P$ value until only $P<0.05$ predictors remained.

This field study was performed with approval of the Flemish government (derogation number ANB/BL/FF-V15-00015).

Animals were handled while wearing a fresh pair of non-powdered, disposable gloves. Equipment and field clothing were cleaned and disinfected between visits to sampling locations.

Detection of chytrid associated disease in the study region was done by postmortem examination of field cases of amphibian disease or mortality over a period of 4 years. Wildlife Health Ghent hosts an amphibian disease hotline where suspect cases of infectious disease are submitted (cases with obvious traumatic causes such as predation or traffic are not withheld). The dead amphibians are routinely examined for the presence of $B d, B s a l$ and Ranavirus using (q) $P_{C R}^{46-48}$

Isolate collection. Isolates were obtained from wild amphibians collected July 2015-September 2016 in the Flanders region of Belgium. Isolation was carried out using the protocol described in Fisher et al. ${ }^{49}$. Using a sterile needle, small skin sections 1-2 mm were cleaned of surface-contaminating bacteria and fungi by dragging it through agar-medium. Subsequently, the tissue sample was transferred into a sterile TGhL medium with antibiotics $(200 \mathrm{mg} / \mathrm{L}$ penicillin-G and $400 \mathrm{mg} / \mathrm{L}$ streptomycin sulphate) and incubated at $20^{\circ} \mathrm{C}$. Six isolates were genotyped and their topology depicted in the global phylogeny in O'Hanlon et al. ${ }^{1}$. These isolates belong to three clades. Full information on each isolate can be found in (Supplementary Table 4). All isolates were preserved in liquid nitrogen at passage 10 . Before use in an experiment, isolates were passaged one time in TGhL broth at $20^{\circ}$ C.

Hygiene and biosafety protocols. The animal experiments were performed under strict BSL2 conditions. During the fieldwork, each individual was handled with a new pair of nitrile gloves. At the end of each field visit, boots, dipnets, funnel traps and other equipments were disinfected with a $1 \%$ Virkon ${ }^{\circledR}$ solution for at least 5 $\min ^{50}$.

Phenotypic characterisation of local Bd isolates. All cultures (BdBE1, 2, 3, 4, 5, $6,7,8,9,10$ and $B d$ JEL423) were grown on TGhL agar at $20^{\circ} \mathrm{C}$, for 5 days. Plates were washed with sterile distilled water, and the water containing the zoospores was passed over a sterile mesh filter with pore size $10 \mathrm{~mm}$ (Pluristrainer, PluriSelect). Using a haemocytometer and inverted microscope (Nikon Eclipse TS100, Nikon) the spores were counted. $B d$ spores $\left(1 \times 10^{6}\right.$ spores per well $)$ were seeded in TGhL medium ( $1.6 \%$ tryptone, $0.4 \%$ gelatin hydrolysate and $0.2 \%$ lactose in $\mathrm{H}_{2} \mathrm{O}$ ) supplemented with $50 \% \mathrm{H}_{2} \mathrm{O}$ into 24 -well tissue culture plates. Wells were photographed using an Olympus CKX41 with an attached camera (Olympus SC50, Olympus) with a $(2560 \times 1920)$ pixel field, $\sim 72,96,120,144$ and $168 \mathrm{~h}$ after inoculation. The experiment was performed in quadruplicate. Images were analysed using ImageJ $1.52 \mathrm{~d}$ software, with the following measurements taken: (1) $\mathrm{N}_{\text {ZOOSPORE }}=$ number of spores in the central $1000 \times 1000$ pixels of the well per image, (2) $\mathrm{N}_{\mathrm{SPOR}}=$ number of sporangia in the central $1000 \times 1000$ pixels, (3) $\mathrm{A}_{\mathrm{SPOR}}=$ area of the largest 10 sporangia, (4) $\mathrm{A}_{\mathrm{ZOOSPORE}}=$ area of 10 random 
spores. To measure fecundity, at day 7 , the following formula was used ((average $\mathrm{N}_{\text {ZOOSPORE }}$ /average $\mathrm{N}_{\mathrm{SPOR}}$ )/average $\mathrm{A}_{\mathrm{SPOR}}$ ) as described in Fisher et al. ${ }^{26}$.

The number of zoospores and the number of sporangia were modelled using generalized linear models (GLM) with Poisson error distributions, while the area of sporangia and the calculated fecundity were modelled using linear models, all with isolate as the response variable. For the area of sporangia, a random effect at the replicate level was included to account for pseudo-replication. The significance of pairwise differences among isolates was assessed using Tukey's HSD test. In addition to differences among isolates for individual characteristics, their overall similarity was assessed using two-dimensional non-metric multidimensional scaling (NMDS). NMDS maps the relationship between the dissimilarity matrix (Bray-Curtis index) to locate each sample along two coordinates in the ordination space $^{51}$. All analyses were performed in $\mathrm{R}^{52}$

Infection trials. The animal experiments were performed with the approval of the ethical committee of the Faculty of Veterinary Medicine (Ghent University EC2016/20, EC2015/86). Only captive-bred animals were used in standardized experiments, using identical environmental conditions that allow comparison of isolate virulence and host susceptibility. All animals were housed individually in terraria at $15{ }^{\circ} \mathrm{C}$ on moist tissue with access to a hiding place. All animals (males/ females) were clinically healthy and derived from breeding colonies that are free of $B d, B s a l$ and Ranavirus as assessed by sampling the skin using cotton-tipped swabs and subsequent performing $\mathrm{qPCR}$ or $\mathrm{PCR}^{46,47,53}$. Individuals were randomly assigned to treatments. All animals were clinically inspected daily. Skin sampling was done weekly and the swabs were analysed for the presence of $B d$ using QPCR described by Hyatt et al. ${ }^{53}$ with the respective isolate used as standard. Sample analysis was blinded. Animals were euthanized by an overdose of pentobarbital. Humane endpoints were set at the loss of self-righting ability and/or change in posture.

\section{Susceptibility of native amphibians to BdGPL isolates under standardized} laboratory conditions. Five captive-bred individuals of seven species (fire salamander (Salamandra salamandra), common spadefoot (Pelobates fuscus), natterjack toad (Epidalea calamita), common toad (Bufo bufo), alpine newt (Icthyosaura alpestris), great crested newt (Triturus cristatus) and European tree frog (Hyla arborea)) were exposed to the local $B d \mathrm{GPL}$ isolate $B d \mathrm{BE} 1$ or the hypervirulent isolate $B d \mathrm{JEL} 423$. In addition, two midwife toads (A. obstetricans) were included as $B d$ JEL423-infected positive control group (the limited number of midwife toads in this experiment were merely included as positive controls, extensive experiments in this species were conducted separately). For inoculum, isolates were grown on TGhL agar at $20^{\circ} \mathrm{C}$, for 5 days. Plates were washed with sterile distilled water, and the water containing the zoospores was passed over a sterile mesh filter with pore size $10 \mathrm{~mm}$ (Pluristrainer, PluriSelect), then counted using a haemocytometer and inverted microscope (Nikon Eclipse TS100, Nikon). Spores were resuspended at a concentration of $1 \times 10^{6}$ spore $\mathrm{ml}^{-1}$. Individuals were inoculated with $1 \mathrm{ml}$ of $1 \times$ $10^{6}$ fresh spores, in separate Petri dishes and placed in climate-controlled $\left(\sim 15^{\circ} \mathrm{C}\right.$ $\& \sim 80 \%$ relative humidity) room for $24 \mathrm{~h}$. Individuals were then transferred to individual plastic containers containing damp tissue and a hide. Small crickets were given ad libitum, providing a constant food supply. Clinical examination of the animals was carried out daily, and tissue replacement and swabbing weekly. Species that did not show infection for over 3 weeks ( 3 weeks negative in qPCR and no clinical signs) in any individual were considered uninfected and removed from experiment at week 8 . Absence of $B d$ infection was confirmed by histopathology and immunohistochemistry. Sample analysis was blinded. We compared the proportion of individuals of each species becoming infected from either isolate using a generalized linear model with binomial error distribution.

Differential BdGPL isolate pathogenicity for midwife toads. Four local phenotypically and genotypically ${ }^{1}$ different isolates originating from different species (Fig. 1, Supplementary Table 5) (BdBE1, $B d \mathrm{BE} 3, B d \mathrm{BE} 4$ and $B d \mathrm{BE} 5)$ and the hypervirulent isolate $B d$ JEL423 were selected to inoculate midwife toads ( $A$. obstetricans). This species is used as model species for susceptibility to virulent $B d$. Negative control animals were sham inoculated with water. Thirty-six newly metamorphosed A. obstetricans were randomly assigned to groups of six for each treatment. Individuals were exposed to $1 \mathrm{ml}$ of $1 \times 10^{6}$ of fresh spores, or distilled water (prepared as previously) for $24 \mathrm{~h}$. Individuals were then transferred to individual plastic containers containing damp tissue and a hiding place in a climate-controlled $\left(\sim 15^{\circ} \mathrm{C} \& \sim 80 \%\right.$ relative humidity) room. Small crickets were given ad libitum, providing a constant food supply. Clinical examination of the animals was carried out daily, and tissue replacement and swabbing weekly. From day 15 onwards, animals were weighed weekly. Individuals that died during the trial or were euthanized due to reaching a humane end point (see above) had skin samples taken for histology and DNA extraction for qPCR. Since after the first inoculation none of the toadlets became positive for $B d B E 5$, in week 5 , an additional five $B d B E 5$ and three $B d J E L 423$ individuals were inoculated and added to the experiment. Sample analysis was blinded.

The probability of an individual dying as a result of infection with different isolates was modelled using a binomial GLM. Penalized regression (package $\mathrm{brglm} 2$ in $\mathrm{R}^{54}$ ) was used to account for perfect separation in some groups, i.e. where all or no animals died within a given treatment group. Since death occurred for all $B d J E L 423$-infected animals and for only one of the animals infected with the local isolates, parametric survival regression model could not be fitted to estimate the mortality rate over time. The relationship between probability of death and the infection load sustained was modelled using a binomial penalized GLM (as above). Infection load was always modelled as the $\log _{10}$ of the genomic equivalent. Mean, maximum and total load over the course of infection were all assessed as predictors.

\section{Protective capacity of low-virulence BdGPL against hypervirulent BdGPL}

challenge in midwife toads. After completion of the multi-isolate A. obstetricans infection trial, all remaining individuals were exposed to $1 \mathrm{ml}$ of $1 \times 10^{6}$ of fresh spores of $B d$ JEL423 (prepared as previously) for $24 \mathrm{~h}$. Individuals were then transferred to individual plastic containers containing damp tissue and a hiding place in a climate-controlled $\left(\sim 15^{\circ} \mathrm{C} \& \sim 80 \%\right.$ relative humidity) room. Small crickets were given ad libitum, providing a constant food supply. Clinical examination of the animals was carried out daily, and tissue replacement and swabbing weekly. Sample analysis was blinded.

As for the initial infection trials, penalized binomial GLMs were used to estimate whether the probability of death following infection with $B d J$ JLL23 depended on (1) the isolate individuals were exposed to in the initial experiment and (2) the mean/maximum/total infection load sustained during the initial experiment. Parametric survival regression was also used to assess whether the survival rates of $B d J E L 423$-re-infected individuals depended on (1) the isolate they had been exposed to in the initial trial and (2) on the mean load of infection in the initial trial. In both cases, the response variable (death) was modelled using a Weibull distribution while accounting for censoring. We repeated all analysed grouping individuals initially infected with local isolates $B d \mathrm{BE} 1 / B d \mathrm{BE} 3$ and $B d \mathrm{BE} 4 /$ $B d \mathrm{BE} 5$, since these two pairs of isolates exhibited similar characteristics in previous experiments.

To confirm the protective capacity of $B d \mathrm{BE} 3,40$ newly metamorphosed $A$. obstetricans were randomly assigned to two groups and exposed to $1 \mathrm{ml}$ of $1 \times 10^{6}$ of fresh spores of $B d \mathrm{BE} 3$, or distilled water (prepared as previously) for $24 \mathrm{~h}$. Animals were then housed individually in plastic containers containing tissue, a hiding place and water dish in a climate-controlled $\left(\sim 20^{\circ} \mathrm{C} \& \sim 80 \%\right.$ relative humidity) room and examined as previously described. Four weeks later, all animals were exposed to $1 \mathrm{ml}$ of $1 \times 10^{6}$ of fresh spores of $B d$ JEL423 (prepared as previously) for $24 \mathrm{~h}$. Individuals were again transferred to individual plastic containers containing tissue, a hiding place and water dish. Small crickets were given ad libitum, providing a constant food supply. Clinical examination of the animals was carried out daily, and tissue replacement and swabbing weekly. Sample analysis was blinded. The readout for this experiment was the comparison of the proportion of $B d$ infected animals after challenge with the highly virulent $B d$ JEL 423 in each of the two treatment groups. Significance of protection was calculated using two-tailed Fisher's exact test.

Bd growth in amphibian mucosomes. Mucosomes were collected from healthy midwife toads (Alytes obstetricans) and fire salamander (Salamandra salamandra) using the bathing method described by Woodhams et al. ${ }^{55}$. For each species, mucosomes were collected from ten animals (animals were not used in the previous experiments). Briefly, each animal was bathed in a Petri dish with HPLC water for $1 \mathrm{~h}$, the volume of water was calculated according to animal surface ${ }^{56,57}$. The collected mucosome solutions were filtered through a $0.2 \mu \mathrm{m}$ unit filter (Whatman, GE Healthcare Life Sciences) and immediately used in the next steps. Two BdGPL isolates, the hypervirulent $B d$ JEL423 and the local $B d B E 1$ were used in this experiment. For each isolate, spores were collected from culture flasks with sporulating sporangia. The medium was collected and filtered using a sterile filter with pore size $10 \mu \mathrm{m}$ (Pluristrainer, PluriSelect). To achieve the target concentration of $1 \times 10^{6}$ spores per $\mathrm{mL}$, the spore suspension was diluted with TGhL broth. Finally, $100 \mu \mathrm{l}$ of this spore suspension was used to obtain $10^{5}$ spores per well of a 96-well flat-bottom plate (Greiner Bio-One).

In each well, $100 \mu \mathrm{L}$ of the mucosome solution was added to $10^{5}$ spores. As positive control, sterile water was added instead of mucosome and the negative control consisted of heat-killed spores $\left(10 \mathrm{~min}\right.$ at $\left.100^{\circ} \mathrm{C}\right)$. All the samples were performed in triplicate. After incubation at $20^{\circ} \mathrm{C}$ for 5 days, the bottom of each well was scraped with a $100 \mu \mathrm{L}$ tip and the liquid was transferred to a $1.5 \mathrm{ml}$ Eppendorf tube for DNA extraction and $\mathrm{qPCR}^{58}$. Growth of $B d \mathrm{BE} 1$ and $B d \mathrm{JEL} 423$ (corrected GE values) was shown by subtracting the mean GE loads of the negative control from the original GE values. Multiple comparisons on the original values were assessed by a Kruskal-Wallis analysis, followed by pairwise Mann-Whitney U-test with a Bonferroni-corrected $P$ value of 0.017 ( $P$ value $=0.05 / 3$ pairwise comparisons) (SPSS version 26).

BdGPL gene expression. We compared gene expression of a selection of virulence genes as identified by Farrer et al. ${ }^{5}$. Using the RNeasy mini kit (Qiagen), total RNA was isolated from fresh $B d$ spores $\left(2.5 \times 10^{7}\right.$ spores per condition) and $B d$ spores $\left(2.5 \times 10^{7}\right.$ spores per condition) that were incubated for $2 \mathrm{~h}$ at $20^{\circ} \mathrm{C}$ with chytrid negative skin of $A$. obstetricans collected with a skin biopsy punch $(6 \mathrm{~mm}) . B d$ spores were collected by putting sterile distilled water on a culture flask containing mature sporangia. Once the zoospores were released, the water containing the 
zoospores was collected. In order to reduce the percentage of mature cells, the water containing the zoospores was passed over a sterile mesh filter with pore size $10 \mu \mathrm{m}$ (Pluristrainer, PluriSelect). The flow-through was used as the zoospore fraction ( $>90 \%$ purity). All conditions were analysed with $B d$ spores originating from $B d$ JEL423, $B d$ BE1- $B d$ BE10 and they were tested in fourfold (biological replicates $n=4)$. Total RNA $(1 \mu \mathrm{g})$ was reverse transcribed to cDNA with the iScript cDNA synthesis kit (Bio-Rad). The housekeeping genes $\alpha$-centractin, APRT, TUB and Ctsyn1 were included as reference genes ${ }^{59}$. The list of genes and sequences of the primers used for quantitative PCR analysis can be found in Supplementary Table 10. Real-time quantitative PCR reactions were run in triplicate (technical replicates $n=3$ ) and the reactions were performed in $10 \mu \mathrm{l}$ volumes using the iQ SYBR Green Supermix (Bio-Rad). The experimental protocol for PCR (40 cycles) was performed on a CFX384 RT-PCR cycler (Bio-Rad) and data were analysed using the Bio-Rad CFX manager 3.1. The results are shown as fold changes of mRNA expression relative to the mRNA expression levels in fresh spores. Fold changes were calculated using the cycle threshold $\left(\Delta \Delta C_{\mathrm{T}}\right)$ method, and they were analysed in SPSS version 25 (SPSS Inc., Chicago, IL, USA). Multiple comparisons were assessed by a Kruskal-Wallis analysis, followed by pairwise Mann-Whitney U-tests adjusted for multiple testing with a Benjamini-Hochberg correction $^{60}$, setting an adjusted $P$ value of 0.05 as significant. Correlation between the relative expression of each isolate to $B d$ JEL 423 for CRN-like genes of fresh spores exposed to midwife toad skin and colonisation capacity from the individuals from the multi-isolate A. obstetricans infection trial was assessed by Spearman's rank correlation in $\mathrm{R}^{54}$.

In vitro infection of $\mathbf{A} \mathbf{6}$ cells. Here, we compared the invasive capacity between hypervirulent and low-virulence local $B d G \mathrm{GL}$ isolates using a cell culture model. The Xenopus laevis kidney epithelial cell line A6 (ATCC-CCL 102) was grown in $75 \mathrm{~cm}^{2}$ cell culture flasks and maintained in complete growth medium (74\% NCTC 109 medium, $15 \%$ distilled water, $10 \%$ fetal bovine serum (FBS) and $1 \%$ of a 10,000 $\mathrm{U} \mathrm{ml}^{-1}$ penicillin-streptomycin solution $(\mathrm{P} / \mathrm{S})$ ) and the cells were incubated at $26^{\circ} \mathrm{C}$ and $5 \% \mathrm{CO}_{2}$ until they reached confluence. Using trypsin, the cells were detached, washed with $70 \%$ Hanks' Balanced Salt Solution without $\mathrm{Ca}^{2+}, \mathrm{Mg}^{2+}$ (HBSS-) by centrifugation for $5 \mathrm{~min}$ at $1500 \mathrm{rpm}$ and resuspended in the appropriate cell culture medium for invasion assays, which were performed as described in Verbrugghe et al. ${ }^{61}$. To assess the germ tube formation, A6 cells were stained with $3 \mu \mathrm{M}$ CellTracker ${ }^{\mathrm{TM}}$ Green CMFDA, seeded ( $10^{5}$ cells per well) in 24well tissue culture plates containing collagen-coated glass coverslips and they were allowed to attach for $2 \mathrm{~h}$ at $20^{\circ} \mathrm{C}$ and $5 \% \mathrm{CO}_{2}$. After washing three times with $70 \%$ HBSS+, they were inoculated with $B d$ zoospores in invasion medium, at a MOI of 1:10. Two hours p.i., the cells were washed three times with $70 \%$ HBSS + and the invasion medium was replaced by staining medium. Four hours p.i., the infected cells were washed three times with HBSS + and they were incubated with Calcofluor White stain $\left(1 \mu \mathrm{g} \mathrm{ml}^{-1}\right.$ in $70 \%$ HBSS +$)$ for $10 \mathrm{~min}$. After washing three times with $70 \%$ HBSS+, the cells were fixed, mounted and analysed using fluorescence microscopy. To assess the invasive growth, A6 cells were seeded and inoculated with $B d$ zoospores as described above. Two days p.i., the infected cells were stained with $3 \mu \mathrm{M}$ CellTracker ${ }^{\mathrm{TM}}$ Green CMFDA, washed three times with $70 \%$ HBSS+ and they were incubated with Calcofluor White stain $\left(10 \mu \mathrm{g} \mathrm{ml}^{-1}\right.$ in $70 \%$ HBSS+) for $10 \mathrm{~min}$. After washing three times with HBSS+, the infected cells were fixed, permeabilized for $2 \mathrm{~min}$ with $0.1 \%$ triton and incubated for $60 \mathrm{~min}$ with a polyclonal antibody against $B d(1 / 1000)$, which was obtained by immunizing rabbits with $B d$-antigen ${ }^{62}$. After washing three times with $70 \%$ HBSS+, the samples were incubated with a goat anti-rabbit Alexa Fluor 568 (1/500). After an incubation of 1 $\mathrm{h}$, the samples were washed three times with $70 \%$ HBSS + , mounted and analysed using fluorescence microscopy and Leica Application Suite (LAS) software X. The Alexa Fluor 568 targeting $B d$ and Calcofluor White stainings are used in concert to assess the ability of $B d$ to penetrate the host cell. Calcofluor White is not internalized by A6 cells, whereas the Alexa Fluor 568 targeting $B d$ staining was applied after permeabilisation of the host cells. As such, intracellular $B d$ will only be targeted by the Alexa Fluor 568 and extracellular $B d$ bodies will be bound with both the Alexa Fluor 568 and Calcofluor White stain. Overlay pictures were made with ImageJ $1.52 \mathrm{~d}$ software. To assess the in vitro infection dynamics of different $B d \mathrm{GPL}$ isolates, three independent in vitro experiments were conducted with every condition being tested in triplicate, with similar results.

Whole-genome sequence analysis. WGS read data were downloaded from NCBI Bioproject PRJNA413876 (SRA sample accession numbers: BdBE1; SRA: SRS2757215, $B d$ BE3; SRA: SRS2757203, $B d$ BE4; SRA: SRS2757202, $B d B E 5$; SRA: SRS2757217, BdJEL423; SRA: SRS2757141). Reads were aligned to the reference $B d$ JEL423 assembly (BioProject PRJNA13653) using BWA mem version 0.7.1763 and the aligned bam files were processed using Picard tools version 2.21 .1 (http:// picard.sourceforge.net/) AddOrReplace, MarkDuplicates, SortSam, Create-

SequenceDictionary and ReorderSam. Variants were called using GATK v4.1.4.0 $0^{64}$ HaplotypeCaller, the output GVCFs were combined using CombineGVCFs, genotyped using GenotypeGVCFs, separated into SNP and Indel variants for filtering using SelectVariants and filtered using VariantFiltration with filters $\mathrm{QD}<2.0 \| \mathrm{FS}$ $>60.0\|\mathrm{MQ}<40.0\|$ MQRankSum $<-12.5||$ ReadPosRankSum $<-8.0$ for SNPs and $\mathrm{QD}<2.0|| \mathrm{FS}>200.0||$ ReadPosRankSum $<-20.0$ for Indels. Variants were annotated using SNPeff v4.365 and the BdJEL423 reference protein annotation
(BioProject PRJNA13653), with those variants annotated as HIGH impact on function used for further analysis. Variants that were only present in all local isolates or only present in the BdJEL423 isolate were identified as the genes of interest for the virulence/local phenotype. Similarly, variants that were only present in $B d \mathrm{Be} 1, B d \mathrm{Be} 3$ and $B d J \mathrm{EL} 423$ while absent in $B d \mathrm{Be} 4$ and $B d \mathrm{Be} 5$, or variants present in $B d \mathrm{Be} 4$ and $B d \mathrm{Be} 5$ while absent in $B d \mathrm{Be} 1, B d \mathrm{Be} 3$ and $B d$ JEL423 were identified as variants of interest for the invasive/epibiotic phenotype. The BdJEL423 reference protein annotation was further annotated using SignalP 4.0 server $^{66}$, the TMHMM Server $2.0^{67}$ and the HMMER 3.2.1 server ${ }^{68}$ using the gathering threshold. Candidate Crinkler proteins were identified using Blastp (BLAST+ 2.9.0 ${ }^{69}$, all candidates listed displayed $100 \%$ identity and e-value of 0 . PFAM domain enrichment in variant-effected groups was identified with right-tailed Fisher's exact test using $q$-value False Discovery Rate. Copy number variation comparisons were made using $\mathrm{CNVkit}^{70}$ version 0.9 .5 batch, segment, scatter, heatmap (with - $\mathrm{d}$ flag to de-emphasize low-amplitude segments) and genemetrics commands, utilising the JEL423 reference assembly and annotation, specifying the $B d$ JEL423 read aligned bam as the normal control compared to the local isolate samples.

Protective capacity of low-virulence BdGPL against highly virulent Bsal in three urodele species. Clinically healthy, $B d, B s a l$ and ranavirus free fire salamanders (27), ribbed newts (Pleurodeles waltl) (24) and marbled newts (Triturus marmoratus) (20) were divided ad random in three treatment groups (every animal was housed individually). Two groups of each species were exposed to local $B d G \mathrm{GP}$ ( $1 \mathrm{ml}$ of $B d \mathrm{SP} 1110^{6}$ spores for $24 \mathrm{~h}$ ), 3 weeks later one of these groups to Bsal $(1 \mathrm{ml}$ of AMFP18/02 $10^{3}$ spores for $24 \mathrm{~h}$ ). At this 21-day time point, a Bsal control group of each species was exposed to Bsal ( $1 \mathrm{ml}$ of AMFP18/02 103 spores for $24 \mathrm{~h}$ ). All animals were clinically inspected daily. Skin sampling was done weekly and the swabs were analysed for the presence of Bsal using QPCR as described by Blooi et al. ${ }^{46,47}$ with the respective isolate used as standard. Bsal infection was confirmed using histopathology. Sample analysis was blinded. Statistical analyses were carried out using generalized linear models and survival regression, following methods described for $B d$ infection trials above (with treatment group in place of isolate).

Reporting summary. Further information on research design is available in the Nature Research Reporting Summary linked to this article.

\section{Data availability}

All data supporting this study are available in the article and corresponding supplementary information files. Genomic read data are available at NCBI GenBank under Bioproject PRJNA413876 (SRA sample accession numbers: BdBE1; SRA SRS2757215, BdBE3; SRA: SRS2757203, BdBE4; SRA: SRS2757202, BdBE5; SRA: SRS2757217, BdJEL423; SRA: SRS2757141). Reads were aligned to the BdJEL423 assembly available under BioProject PRJNA13653, assembly accession GCA_000149865.1. Source data are provided with this paper.

Received: 6 December 2019; Accepted: 5 October 2020; Published online: 26 October 2020

\section{References}

1. O'Hanlon, S. J. et al. Recent Asian origin of chytrid fungi causing global amphibian declines. Science 360, 621-627 (2018).

2. Scheele, B. C. et al. Amphibian fungal panzootic causes catastrophic and ongoing loss of biodiversity. Science 363, 1459-1463 (2019).

3. Berger, L. et al. History and recent progress on chytridiomycosis in amphibians. Fungal Ecol. 19, 89-99 (2016).

4. Martel, A. et al. Batrachochytrium salamandrivorans sp. nov. causes lethal chytridiomycosis in amphibians. Proc. Natl Acad. Sci. USA 110, 15325-15329 (2013).

5. Farrer, R. A. et al. Genomic innovations linked to infection strategies across emerging pathogenic chytrid fungi. Nat. Commun. 8, 14742 (2017).

6. Schloegel, L. M. et al. Novel, panzootic and hybrid genotypes of amphibian chytridiomycosis associated with the bullfrog trade. Mol. Ecol. 21, 5162-5177 (2012).

7. Bosch, J., Martínez-Solano, I. \& García-París, M. Evidence of a chytrid fungus infection involved in the decline of the common midwife toad (Alytes obstetricans) in protected areas of central Spain. Biol. Conserv. 97, 331-337 (2001).

8. Briggs, C. J., Knapp, R. A. \& Vredenburg, V. T. Enzootic and epizootic dynamics of the chytrid fungal pathogen of amphibians. Proc. Natl Acad. Sci. USA 107, 9695-9700 (2010).

9. Weldon, C., Channing, A., Misinzo, G., \& Cunningham, A. A. Disease driven extinction in the wild of the Kihansi spray toad (Nectophrynoides asperginis). bioRxiv https://doi.org/10.1101/677971 (2019). 
10. Clare, F. C. et al. Climate forcing of an emerging pathogenic fungus across a montane multi-host community. Philos. Trans. R. Soc. Lond. Ser. B Biol. Sci. 371, 20150454 (2016).

11. Bosch, J. \& Martínez-Solano, I. Chytrid fungus infection related to unusual mortalities of Salamandra salamandra and Bufo bufo in the Peñalara Natural Park, Spain. Oryx 40, 84-89 (2006).

12. Bosch, J., Carrascal, L. M., Durán, L., Walker, S. \& Fisher, M. C. Climate change and outbreaks of amphibian chytridiomycosis in a montane area of Central Spain; is there a link? Proc. R. Soc. B Biol. Sci. 274, 253-260 (2007).

13. Van der Sluijs, A. et al. Environmental determinants of recent endemism of Batrachochytrium dendrobatidis infections in amphibian assemblages in the absence of disease outbreaks. Conserv Biol. 28, 1302-1311 (2014).

14. Baláž, V. et al. Assessing risk and guidance on monitoring of Batrachochytrium dendrobatidis in Europe through identification of taxonomic selectivity of infection. Conserv Biol. 28, 213-223 (2013).

15. Kärvemo, S., Meurling, S., Berger, D., Höglund, J. \& Laurila, A. Effects of host species and environmental factors on the prevalence of Batrachochytrium dendrobatidis in northern Europe. PLoS ONE 13, e0199852 (2018).

16. van der Sluijs, A., Canessa, S., Martel, A. \& Pasmans, F. Fragile coexistence of a global chytrid pathogen with amphibian populations is mediated by environment and demography. Proc. R. Soc. B 284, 1864 (2017).

17. Schmeller, D. S. et al. Microscopic aquatic predators strongly affect infection dynamics of a globally emerged pathogen. Curr. Biol. 24, 176-180 (2014).

18. Ellison, A. R. et al. More than skin deep: functional genomic basis for resistance to amphibian chytridiomycosis. Genome Biol. Evolution 7, 286-298 (2014).

19. Ellison, A. R. et al. Fighting a losing battle: vigorous immune response countered by pathogen suppression of host defenses in the chytridiomycosissusceptible frog Atelopus zeteki. G3: Genes, Genomes, Genet. 4, 1275-1289 (2014).

20. Voyles, J. et al. Shifts in disease dynamics in a tropical amphibian assemblage are not due to pathogen attenuation. Science 359, 1517-1519 (2018).

21. Garner, T. W. J., Rowcliffe, J. M. \& Fisher, M. C. Climate, chytridiomycosis or condition: an experimental test of amphibian survival. Glob. Change Biol. 17, 667-675 (2011).

22. Walker, S. F. et al. Factors driving pathogenicity vs. prevalence of amphibian panzootic chytridiomycosis in Iberia. Ecol. Lett. 13, 372-382 (2011).

23. Laking, A. E., Ngo, H., Pasmans, F., Martel, A. \& Nguyen, T. Batrachochytrium salamandrivorans is the predominant chytrid fungus in Vietnamese salamanders. Sci. Rep. 7, 44443 (2017).

24. Farrer, R. A. et al. Multiple emergences of genetically diverse amphibianinfecting chytrids include a globalized hypervirulent recombinant lineage. Proc. Natl Acad. Sci. USA 108, 18732-18736 (2011).

25. Langhammer, P. F. et al. A fungal pathogen of amphibians, Batrachochytrium dendrobatidis, attenuates in pathogenicity with in vitro passages. PLoS ONE 8, e77630 (2013).

26. Fisher, M. C. et al. Proteomic and phenotypic profiling of the amphibian pathogen Batrachochytrium dendrobatidis shows that genotype is linked to virulence. Mol. Ecol. 18, 415-429 (2009).

27. Garner, T. et al. Chytrid fungus in Europe. Emerg. Infect. Dis. 11, 1639-1641 (2005).

28. LÁŽ, V. et al. Assessing risk and guidance on monitoring of Batrachochytrium dendrobatidis in Europe through identification of taxonomic selectivity of infection. Conserv Biol. 28, 213-223 (2014).

29. Hallengren, A. Chytridiomykosis-Ett hot mot svenska groddjur (Länsstyrelsen Skåne, Malmö, 2013).

30. Selin, C., de Kievit, T. R., Belmonte, M. F. \& Fernando, W. G. D. Elucidating the role of effectors in plant-fungal interactions: progress and challenges. Front. Microbiol. 7, 600 (2016)

31. Na Pombejra, S., Jamklang, M., Uhrig, J. P., Vu, K. \& Gelli, A. The structurefunction analysis of the Mprl metalloprotease determinants of activity during migration of fungal cells across the blood-brain barrier. PLoS ONE 13, e0203020 (2018).

32. $\mathrm{Vu}, \mathrm{K}$. et al. Invasion of the central nervous system by Cryptococcus neoformans requires a secreted fungal metalloprotease. mBio 5, https://doi. org/10.1128/mBio.01101-14 (2014).

33. Zepelin, M. B., Beggah, S., Boggian, K., Sanglard, D. \& Monod, M. The expression of the secreted aspartyl proteinases Sap4 to Sap6 from Candida albicans in murine macrophages. Mol. Microbiol. 28, 543-554 (1998).

34. Monod, M. \& Zepelin, M. B. Secreted aspartic proteases as virulence factors of Candida species. Biol. Chem. 383, 1087-1093 (2002).

35. Refsnider, J. M., Poorten, T. J., Langhammer, P. F., Burrowes, P. A. \& Rosenblum, E. B. Genomic correlates of virulence attenuation in the deadly amphibian chytrid fungus, Batrachochytrium dendrobatidis. G3: Genes, Genomes, Genet. 5, 2291-2298 (2015).

36. Pasmans, F., Mutschmann, F., Halliday, T. \& Zwart, P. Amphibian decline: the urgent need for amphibian research in Europe. Vet. J. 171, 18-19 (2006).

37. Rosa, G. et al. Evidence of chytrid-mediated population declines in common midwife toad in Serra da Estrela, Portugal. Anim. Conserv. 16, 306-315 (2013).
38. Castro, F. \& Bolker, B. Mechanisms of disease-induced extinction. Ecol. Lett. 8, 117-126 (2005).

39. Jenkinson, T. S. et al. Globally invasive genotypes of the amphibian chytrid outcompete an enzootic lineage in coinfections. Proc. R. Soc. B Biol. Sci. 285, 20181894 (2018).

40. Greenspan, S. et al. Hybrids of amphibian chytrid show high virulence in native hosts. Sci. Rep. 8, 9600 (2018).

41. Ghosh, P. \& Fisher, M. C. Dr Jekyll and Mrs Hyde: risky hybrid sex by amphibian-parasitizing chytrids in the Brazilian Atlantic Forests. Mol. Ecol. 25, 2961-2963 (2016).

42. Stegen, G. et al. Drivers of salamander extirpation mediated by Batrachochytrium salamandrivorans. Nature 544, 353-356 (2017).

43. Longo, A. V., Fleischer, R. C. \& Lips, K. R. Double trouble: co-infections of chytrid fungi will severely impact widely distributed newts. Biol. Inv. 21, 2233-2245 (2019).

44. McMahon, T. A. et al. Amphibians acquire resistance to live and dead fungus overcoming fungal immunosuppression. Nature 511, 224-227 (2014).

45. Peig, J. \& Green, A. J. New perspectives for estimating body condition from mass/length data: the scaled mass index as an alternative method. Oikos 118 1883-1891 (2009)

46. Blooi, M. et al. Duplex real-time PCR for rapid simultaneous detection of Batrachochytrium dendrobatidis and Batrachochytrium salamandrivorans in amphibian samples. J. Clin. Microbiol. 51, 4173-4177 (2013).

47. Blooi, M. et al. Correction for Blooi et al., Duplex real-time PCR for rapid simultaneous detection of Batrachochytrium dendrobatidis and Batrachochytrium salamandrivorans in amphibian samples. J. Clin. Microbiol. 54, 246-246 (2016).

48. Holopainen, R., Honkanen, J., Jensen, B., Ariel, E. \& Tapiovaara, H. Quantitation of ranaviruses in cell culture and tissue samples. J. Virol. Methods 171, 225-233 (2011).

49. Fisher, M. C. et al. Development and worldwide use of non-lethal, and minimal population-level impact, protocols for the isolation of amphibian chytrid fungi. Sci. Rep. 8, 7772 (2018)

50. Rooij, P., Pasmans, F., Coen, Y. \& Martel, A. Efficacy of chemical disinfectants for the containment of the salamander chytrid fungus Batrachochytrium salamandrivorans. PLoS ONE 12, e0186269 (2017).

51. Minchin, P. R. Theory and models in vegetation science. Proceedings of Symposium, Uppsala, July 8-13, 1985. https://doi.org/10.1007/978-94-0094061-1_9 (1987).

52. Team, C. R. R: A Language and Environment for Statistical Computing (2019).

53. Hyatt, A. et al. Diagnostic assays and sampling protocols for the detection of Batrachochytrium dendrobatidis. Dis. Aquat. Organ 73, 175-192 (2007).

54. Kosmidis, I. brglm2: Bias reduction in generalized linear models. $R$ package version 0.1 .8 (2018)

55. Woodhams, D. C. et al. Interacting symbionts and immunity in the amphibian skin mucosome predict disease risk and probiotic effectiveness. PLOS ONE 9 e96375 (2014)

56. McClanahan, L. \& Baldwin, R. Rate of water uptake through the integument of the desert toad, Bufo punctatus. Comp. Biochem Physiol. 28, 381-389 (1969).

57. Spight, T. The water economy of salamanders: evaporative water loss. Physiol. Zool. 41, 195-203 (1968).

58. Boyle, D., Boyle, D., Olsen, V., Morgan, J. \& Hyatt, A. Rapid quantitative detection of chytridiomycosis (Batrachochytrium dendrobatidis) in amphibian samples using real-time Taqman PCR assay. Dis. Aquat. Organ 60, 141-148 (2004).

59. Verbrugghe, E., Pasmans, F. \& Martel, A. Reference gene validation of Batrachochytrium dendrobatidis and Batrachochytrium salamandrivorans for quantitative real-time PCR studies. Sci. Rep. 9, 18534 (2019).

60. Benjamini, Y. \& Hochberg, Y. Controlling the false discovery rate: a practical and powerful approach to multiple testing. J. R. Stat. Soc. Ser. B Methodol. 57, 289-300 (1995)

61. Verbrugghe, E. et al. In vitro modeling of Batrachochytrium dendrobatidis infection of the amphibian skin. PLoS ONE 14, e0225224 (2019).

62. Thomas, V. et al. Recommendations on diagnostic tools for Batrachochytrium salamandrivorans. Transbound. Emerg. Dis. 65, e478-e488 (2018).

63. Li, H. \& Durbin, R. Fast and accurate long-read alignment with BurrowsWheeler transform. Bioinformatics 26, 589-595 (2010).

64. Van der Auwera, G. A. et al. From FastQ data to high confidence variant calls the Genome Analysis Toolkit best practices pipeline. Curr. Protoc. Bioinforma. 11, 11.10.1-11.10.33 (2013).

65. Cingolani, P. et al. A program for annotating and predicting the effects of single nucleotide polymorphisms, SnpEff: SNPs in the genome of Drosophila melanogaster strain w 1118; iso-2; iso-3. Fly 6, 80-92 (2012).

66. Petersen, T. N., Brunak, S., von Heijne, G. \& Nielsen, H. SignalP 4.0: discriminating signal peptides from transmembrane regions. Nat. Methods 8 785-786 (2011).

67. Krogh, A., Larsson, B., von Heijne, G. \& Sonnhammer, E. L. L. Predicting transmembrane protein topology with a hidden markov model: application to complete genomes. J. Mol. Biol. 305, 567-580 (2001). 
68. Finn, R. D. et al. HMMER web server: 2015 update. Nucleic Acids Res. 43, W30-W38 (2015)

69. Altschul, S. F. et al. Gapped BLAST and PSI-BLAST: a new generation of protein database search programs. Nucleic Acids Res. 25, 3389-3402 (1997).

70. Talevich, E., Shain, A. H., Botton, T. \& Bastian, B. C. CNVkit: genome-wide copy number detection and visualization from targeted DNA sequencing. PLoS Comput. Biol. 12, e1004873 (2016).

\section{Acknowledgements}

This work was funded by the Ghent University Special Research Fund (BOF16-GOA024.08) and the 'Agentschap voor Natuur en Bos' (BL-100/FIN/15-00229). E.V., M.K. and W.B. were supported by the Research Foundation Flanders (FWO grants 12E6616N, 1507119 N, 1111119N, 11ZK916N-18N). Z.L. is supported by the China Scholarship Council (CSC, 201707650012) and Ghent University Special Research Fund (BOF17/ CHN/018). We thank Iwan Lewylle for the field-work assistance. Kim Roelants kindly provided the artwork.

\section{Author contributions}

A.M. and F.P. designed the research. A.M., M.S.G., E.V., M.K., M.B., W.B., N.D.T., Z.L., G.S., R.V.L. and S.V.P. carried out the research. A.M., M.S.G., E.V., M.K., W.B., S. Canessa, L.L., Z.L., D.S., R.V.L. and F.P. analysed the data. M.S.G., E.V., W.B., S. Canessa, S. Carranza, S.C., D.F.G., P.G., L.L., Z.L., D.S., R.V.L., M.V.E., M.V., F.P. and A.M. interpreted the data. A.M., F.P., M.S.G., E.V., M.K., W.B. and S. Canessa wrote the paper with input from all other authors.

\section{Competing interests}

The authors declare no competing interests.

\section{Additional information}

Supplementary information is available for this paper at https://doi.org/10.1038/s41467020-19241-7.

Correspondence and requests for materials should be addressed to A.M.

Peer review information Nature Communications thanks Jamie Voyles and the other, anonymous, reviewer for their contribution to the peer review of this work. Peer reviewer reports are available.

Reprints and permission information is available at http://www.nature.com/reprints

Publisher's note Springer Nature remains neutral with regard to jurisdictional claims in published maps and institutional affiliations.

\begin{abstract}
(c) (i) Open Access This article is licensed under a Creative Commons Attribution 4.0 International License, which permits use, sharing, adaptation, distribution and reproduction in any medium or format, as long as you give appropriate credit to the original author(s) and the source, provide a link to the Creative Commons license, and indicate if changes were made. The images or other third party material in this article are included in the article's Creative Commons license, unless indicated otherwise in a credit line to the material. If material is not included in the article's Creative Commons license and your intended use is not permitted by statutory regulation or exceeds the permitted use, you will need to obtain permission directly from the copyright holder. To view a copy of this license, visit http://creativecommons.org/ licenses/by/4.0/.
\end{abstract}

(C) The Author(s) 2020 